\title{
Disentangling the bulk and exit surface contribution on projectile-charge-state evolution
}

\author{
Prashant Sharma* and Tapan Nandi ${ }^{\dagger}$ \\ Inter-University Accelerator Centre, Aruna Asaf Ali Marg, New Delhi 110067, India
}

(Received 26 February 2018; revised manuscript received 28 January 2019; published 26 March 2019)

\begin{abstract}
Charge state evolution of the projectile ions while traversing through the solid target medium has been explored using the radiative electron capture process. The measured centroid energies of the convoluted radiative electron capture peak structures have been used to determine the mean K-shell binding energies and mean charge state of the projectile ions. It has been observed that the mean charge states of present measurements are lower than the earlier measurements done using the characteristic $\mathrm{K}_{\alpha} \mathrm{X}$-ray transitions. The difference is due to the capture of target electrons in the inner-shell vacancies, created during the collision process, of projectile ions. Further, the measured mean charge states are compared with the empirical predictions. A significant discrepancy between experimental and theoretical values has been observed, which is attributed to the multielectron capture by projectile ions due to nonradiative electron capture process from the exit surface while exiting from the foil. The significant variation between mean charge state values obtained from different tools provides a clear indication of the dynamic nature of the charge-changing mechanism at different regions (entrance surface, bulk, and exit surface) of the ion-solid interaction. The present results can be used to validate the departure between the theory and experiment on the charge state dependent stopping powers.
\end{abstract}

DOI: $10.1103 /$ PhysRevAccelBeams.22.034501

\section{INTRODUCTION}

The study of the charge state evolution of projectile ions traversing solid or gaseous targets has been a subject of great interest for more than 100 years [1-3]. The process is highly intricate because various physical phenomena including ionization, excitation, radiative decay, Auger decay, nonradiative electron capture (NREC), etc., are known to coexist (see a review [4]) until radiative electron capture (REC) was discovered in 1972 [5]. Subsequently, many other processes have been identified such as multiple vacancy creations [6], electron loss to continuum (ELC) [7], electron capture to continuum (ECC) [8], multiple electron capture [9], radiative electron capture in the continuum (RECC) [10], three-electron-Auger process [11], radiative double electron capture (RDEC) [12], etc. It is observed that the complexity in accurate measurements, as well as theoretical estimations of such processes, is more prevalent with the solid targets than the gaseous targets due

\footnotetext{
*Present address: Faculty of Physics, Weizmann Institute of Science, Rehovot 7610001, Israel.

phyprashant@gmail.com

nanditapan@gmail.com

Published by the American Physical Society under the terms of the Creative Commons Attribution 4.0 International license. Further distribution of this work must maintain attribution to the author(s) and the published article's title, journal citation, and DOI.
}

to the multiple collisions during the interactions. Several physical processes originating from the solid surfaces, for example, wake riding electron capture [13] leading to the formation of circular Rydberg states [14], electron capture in low-lying and high-lying states, etc., contribute in the dynamicity of the charge-changing processes. The excited states produced by any of the above processes can be further affected by the surface energy loss field (SELF) at the exit surface [15]. These charge-changing processes can be investigated through the distribution of charge state fractions (CSF) called charge state distribution (CSD) for both experiments and theories. Several extensive reviews on CSDs can be found in the literature including that of Allison [16], Betz [17], Wittkower and Betz [18], Shima et al. $[19,20]$. These reviews provide a crucial theoretical background in terms of the data collection to date. The CSDs are normally measured by the standard electromagnetic techniques [21-24] and in order to understand the measured equilibrium charge state data, many empirical formulas such as Thomas-Fermi model, Bohr model, Betz model, Nikolaev-Dmitriev model, To-Drouin model, Shima-Ishihara-Mikumo model, Itoh model, ZieglerBiersack-Littmark model, Schiwietz model, etc., have been developed in tune with the experimental results from electromagnetic measurements [ 25 and references therein]. Interestingly, in some distinct cases, like, calculation of non-equilibrium charge state distribution [26,27], estimation of equilibrium target thickness [28], etc., the empirical 
predictions readily fail to estimate desired parameters. The contribution of the charge-changing processes in the bulk is further modified by the charge-exchange phenomena from the exit surface of the foil. Whatsoever, this does not pose any problem to the electromagnetic technique based measurements as it accounts for the charge state of the ions irrespective of excitation state and the underlying mechanisms. However, for a theory, all the physical processes occurring at the bulk and surface of the foil need to be considered explicitly.

It is worth mentioning that the contribution from electron capture process can come from both the bulk and surface. Whereas the wake riding electron [13] driven processes at the exit surface gives rise to excited states are either shortlived low lying states or long-lived Rydberg states including the circular Rydberg states [14]. Further, wake and dynamic screening effect $[29,30]$ on the levels so produced in the bulk or the surface cannot be ignored too. Hence, disentangling the charge-changing processes and chargeexchange phenomena cropping up in the bulk and at the surface of the target, respectively, is extremely difficult and there is no way that the electromagnetic methods can differentiate between the said processes. Interestingly, $\mathrm{X}$-ray spectroscopy technique is convenient in this respect and can measure CSDs quite efficiently. In our earlier work [31], the x-ray spectroscopy technique has been employed to measure the CSD and its various parameters like mean charge state, distribution width, etc., using the characteristic $\mathrm{K}$ x-rays. Further, by comparing the experimental [31-33] and empirically [34] predicted mean charge states, we have portrayed a picture of disentanglement of the bulk effects from the total effect of the bulk and surface. In the present work, we contemplate REC x-ray peak structures to obtain the mean charge states for an intermediate stage, where the charge-changing processes are occurring in the bulk in addition to the charge-exchange contribution from REC process that occurs at the bulk as well as the surface.

\section{EXPERIMENTAL PROCEDURE}

The experiments were performed with the 15 UD Pelletron accelerator facility at IUAC, New Delhi, India. Well-collimated heavy ion beams of ${ }^{56} \mathrm{Fe}^{q+}(q=7-12)$ and ${ }^{58} \mathrm{Ni}^{q+}(q=7-13)$ in the energy range of $1.65-3.03 \mathrm{MeV} / \mathrm{u}$ were bombarded on the self-supporting carbon foil target of thickness $80 \mu \mathrm{g} / \mathrm{cm}^{2}\left(\approx 113 \mu \mathrm{g} / \mathrm{cm}^{2}\right.$ as the target is placed at $45^{\circ}$ to the beam axis). The target thickness was appropriately chosen such that the projectile ions with the highest beam energy can also attain the equilibrium charge state distribution. The experiments were performed with the Low Energy Germanium Detector (GUL0035, Canberra Inc.), equipped with $25 \mu \mathrm{m}$ thick Be entrance window and having resolution $150 \mathrm{eV}$ at $5.9 \mathrm{keV}$ in the ideal conditions and constant quantum efficiency in the range of $5-20 \mathrm{keV}$ ). The detector was placed at $90^{\circ}$ to the beam axis to minimizing the Doppler shift for the projectile x-rays. Using appropriate collimators, the $\mathrm{x}$-rays observed by the detector was restricted to the ion-solid interaction zone or the prompt $\mathrm{X}$-rays only. The detector was calibrated by ${ }^{57} \mathrm{Co}$ and ${ }^{241} \mathrm{Am}$ standard radioactive sources. The resolution was found to be about $200 \mathrm{eV}$ at $6.4 \mathrm{keV}$ with the experimental conditions prevailing in the beam hall. Further, in the case of ${ }^{58} \mathrm{Ni}$ beam, the in-beam calibration with $\mathrm{Fe} K_{\alpha}$ peak due to the beam halo hitting the carbon foil holder made of stainless steel was also done. However, in the case of ${ }^{56} \mathrm{Fe}$ beam experiment, the beam halo was minimized by passing the beam through a blank target frame so that its presence did not affect the characteristic projectile peak structures. Using an appropriate reentry cup, the $\mathrm{x}$-ray detector was inserted into the chamber separated by a thin Mylar window of $6 \mu \mathrm{m}$ at the interface. The beam was collected in the secondary electron suppressed Faraday cup. Two solid surface barrier detectors were used at $\pm 10^{\circ}$ to monitor the beam direction. The vacuum chamber was maintained at a pressure around $1 \times 10^{-6}$ Torr throughout the experiment.

\section{RADIATIVE ELECTRON CAPTURE: THEORY AND PRESENT METHOD}

During the passage of the projectile ions through the target foil, the interactions lead to the multiple vacancies in various shells of both the atomic systems. The short lifetime of inner shell vacancies (few fs) compared to the transit time of projectile ion through the target foil (few tens of fs), causes a complex chain of collision events resulting in a composition of various projectile charge states. The vacancies so created are filled by different relaxation processes, e.g., characteristic x-ray emissions, Auger emission, REC process, NREC process, etc. These relaxation processes vary from system to system, for instance, the Auger process is predominant in low- $Z$ elements, whereas X-ray emission processes (REC and characteristic $\mathrm{x}$-ray emissions) dominate over Auger emission in high- $Z$ elements. Whatsoever, after a significant number of collisions, the charge-changing processes reach to a certain balance, and subsequently, an equilibrium charge state distribution is established. The mean of the charge states attained at such equilibrium is termed as the mean charge state of the corresponding atomic systems. It is noteworthy that the x-ray emissions from these individual states cannot be resolved with the detectors used in the present work, and therefore we have observed a convolution of the x-ray transitions from all the projectile charge states so evolved.

In the present work, we have used the REC peak structures to estimate the mean charge state of the projectile ions. The REC process, the capture of quasi-free valence electrons by the projectile ions from the target atoms accompanied by the spontaneous $\mathrm{x}$-ray emissions, has been extensively studied with the bare projectile ions, where the electron capture process can only occur among the various charge-changing processes. In some studies, $\mathrm{H}$-like projectile ions have also been used to investigate the 
REC process as here the ionization is limited and mostly the electron capture reigns. Note that multielectron projectile can provide a better ground for exploring the REC process in the K-shell followed by K-shell ionization processes. However, limited work has been done involving the multielectron projectile ions [35-37].

In the nonrelativistic case, REC photon energy in the projectile reference frame is given by [38],

$$
E_{\mathrm{REC}}=B_{P}-B_{T}+\frac{m_{e}}{M_{p}} E_{k}+\vec{v} \cdot \vec{p}
$$

where $B_{T}$ and $B_{P}$ are the binding energy of the electron in the initial state of target atom and final state of the projectile atom, whereas $m_{e}, M_{p}$, and $E_{k}$ are the electron mass, projectile mass, and projectile energy, respectively. The symbols $v$ and $p$ represent the projectile ion velocity and target electron momentum, respectively. The full width at to bare or $\mathrm{H}$-like projectile ion is given as,

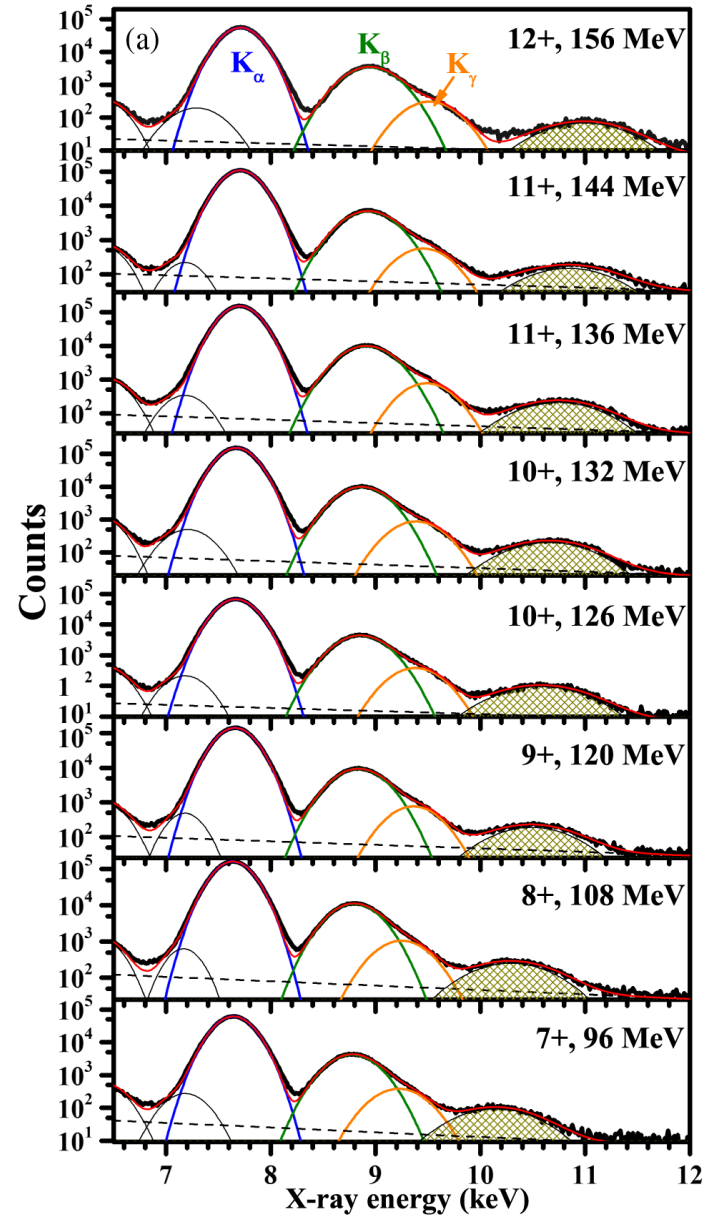
half maximum (FWHM) of the REC x-rays corresponding

$$
\mathrm{FWHM}=\triangle E_{\mathrm{REC}}=2 v_{0} p_{z}=2\left[4\left(m_{e} / M_{p}\right) E_{k} B_{T}\right]^{1 / 2}
$$

where the factor of 2 is due to the fact that in the target atom, the orbiting electron has two directions of initial momentum, which contributes twice in the width of the K-REC peak [39].

The REC peak structure contains useful information related to both the target and projectile atomic systems and thus can be used as a sensitive probe to explore the dynamics of charge-changing processes during the ionsolid collisions. For example, the shape of the REC peak defines the momentum distribution of electrons in the target atom as seen in Eq. (2), whereas having known the projectile energy and initial state binding energy, the observed K-REC x-ray energy allows the explicit measurement of mean K-shell binding energies of the projectile ions [40]. Further, as the mean K-shell binding energies correspond to the electronic structure of the atomic system, one can also determine the projectile mean charge state during the passage from the target medium.

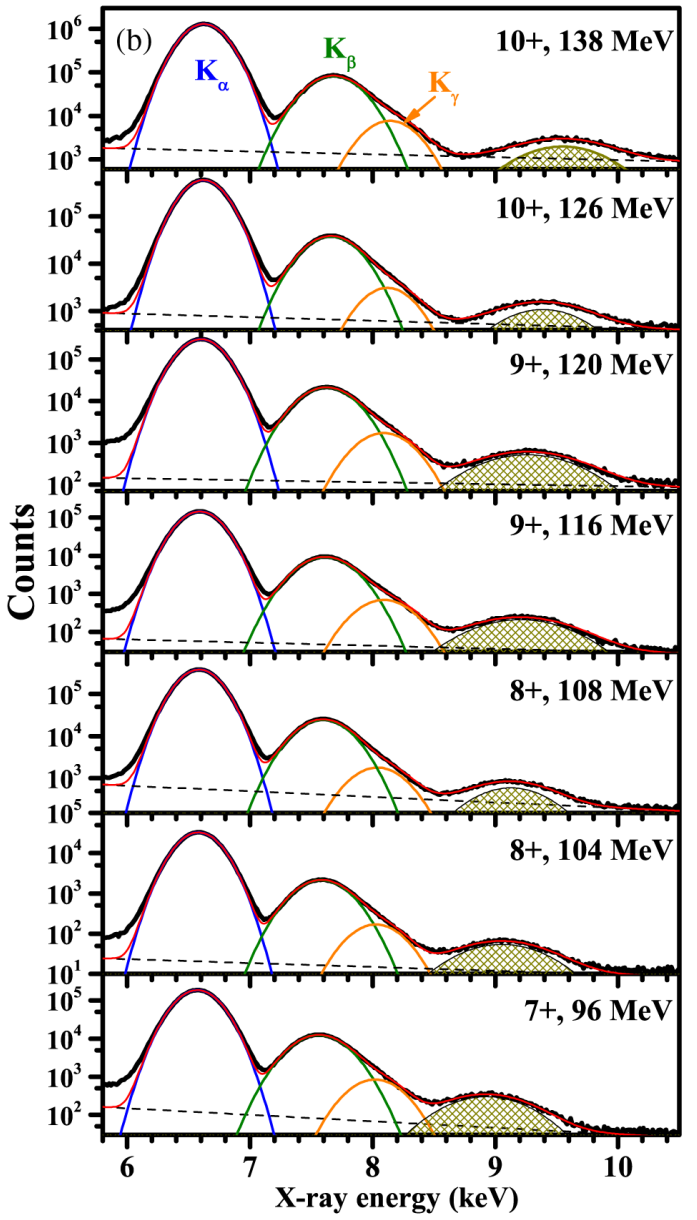

FIG. 1. X-ray spectra for (a) ${ }^{58} \mathrm{Ni}^{q+}(q=7-13)$ and (b) ${ }^{56} \mathrm{Fe}^{q+}(q=7-10)$ beam on $80 \mu \mathrm{g} / \mathrm{cm}^{2}\left(\approx 113 \mu \mathrm{g} / \mathrm{cm}^{2}\right.$ at $45^{\circ}$ to the beam axis) carbon foil at different beam energies. The first three peaks represent the characteristic X-ray transitions $\left(\mathrm{K}_{\alpha}, \mathrm{K}_{\beta}\right.$, and $\left.\mathrm{K}_{\gamma}\right)$, whereas the highlighted peaks are the radiative electron capture structures. The dashed lines represent the exponential continuum background. 


\section{RESULTS AND DISCUSSION}

The observed $\mathrm{x}$-ray spectra, displaying four major peak structures, for all the beam energies have been shown in Fig. 1. The first three peaks represent the characteristic $\mathrm{K}$ $\mathrm{x}$-ray transitions $\mathrm{K}_{\alpha}, \mathrm{K}_{\beta}$, and $\mathrm{K}_{\gamma}$, whereas the fourth broad peak belongs to the K-REC structure. It is observed that the raw spectra obtained at different beam energies have no pile-up contributions at the concerned $\mathrm{x}$-ray region, i.e., K-REC position. Further, the overall $x$-ray count rate remains less than $1 \mathrm{k} \mathrm{Hz}$ to restrict pile up contribution in the spectra. Therefore, with this low count rate, the deadtime correction is less than $1 \%$ for the $\mathrm{x}$-ray intensities [41].

In the present experiment, low charge states $\left(7^{+}-12^{+}\right)$ used with the $\mathrm{Fe}$ and $\mathrm{Ni}$ ion beams ascertain lack of $\mathrm{K}$-vacancy. Thus, the mechanisms of the characteristic or K-REC x-ray emissions necessarily involve K-excitation or K-ionization initially, and the cross-section of such events is high for heavy ion-induced ion-solid collisions. Subsequently, a few of these K-vacancies are filled by either REC or NREC process. In our earlier work, the $\mathrm{K}_{\alpha}$ peak structure has been used to obtain the CSD produced at the bulk only during the ion-solid collisions, which excludes the atomic processes leading to REC, metastable and any high Rydberg states that may also occur at the bulk [31]. The X-ray peak relevant to the REC process in the present experiment is either K-REC or L-REC. The K-REC appears at higher energy side and the L-REC in the lower energy side of the $\mathrm{K}_{\alpha}$ peak. Thus, in the present experimental condition, they are well resolved in the spectra. Transition probabilities of x-ray emissions from the metastable as well as high Rydberg states are much smaller than that of the $\mathrm{K}_{\alpha}$ line. Hence, they hardly show any significant existence in the prompt $\mathrm{x}$-ray spectra containing the $\mathrm{K}_{\alpha}, \mathrm{K}_{\beta}$, and $\mathrm{K}_{\gamma}$ transitions in addition to the K-REC peak, which does not have any lifetime structure at all. Here we note that the ion-solid collisions lead to creating the vacancy in the $\mathrm{K}$-shell of the projectile ions, that can be filled either by the electrons from the higher shells of the same projectile ions (inner-shell transitions) or the electrons of the target atoms in the bulk and at the surface of the target (REC and NREC process). In the present work, we focus only on the K-REC peak to obtain the CSD, which thus accounts for all the atomic processes occurring at the bulk responsible for the creation of K-shell vacancies along with the REC contributions arising from the bulk as well as the surface of the foil. A schematic diagram illustrating the various stages of charge state evolution of projectile ions during the passage from target medium has been shown in Fig. 2. Note again that whatever small part of atomic processes going to the metastable and high Rydberg states cannot interfere at all with the K-REC because of time structures; K-REC has no lifetime, and the metastable and high Rydberg states have a long lifetime.

It is noteworthy that the structure of the REC profile depends on various factors like detector resolution, Doppler broadening, CSD evolved during the ion-solid collisions, etc. Therefore, the measured FWHM is corrected for the detector resolution and the Doppler broadening, and then the values so obtained are compared with the theoretical values using Eq. (2). The resultant effect of these corrections leads to a reduction of the measured value of FWHMs by about $10-15 \%$. Whereas, the other corrections, i.e., Doppler broadening due to the finite size of the collimators and detector area, are quite small and can be neglected. For evaluating the final error for the FWHM data, we have conceded the error as a combination of the systematic and

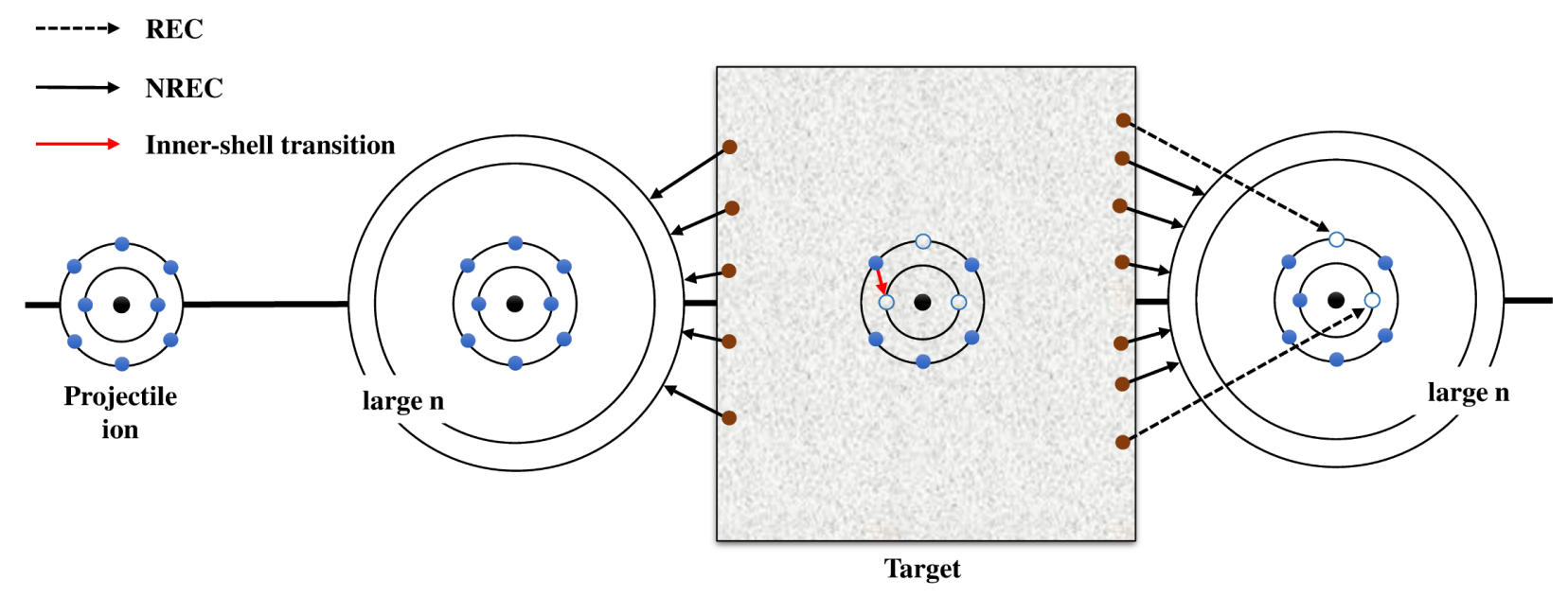

FIG. 2. Illustration of different stages of the charge state evolution of projectile ions during the passage of the projectile ions through target medium. The approaching highly charged projectile ion firstly captures some electrons from the entrance surface in the high-lying Rydberg states (NREC process). During the passage from bulk, these loosely bound electrons including some inner-shell electrons cannot survive and get stripped from the corresponding projectile ion. The inner-shell vacancies so created can be filled either by the inner-shell transitions or charge-exchange processes. While exiting from the target, the projectile ion may capture some electrons from the exit surface into the high-lying Rydberg states (NREC process) as well as in the vacant inner-shells (REC process). 

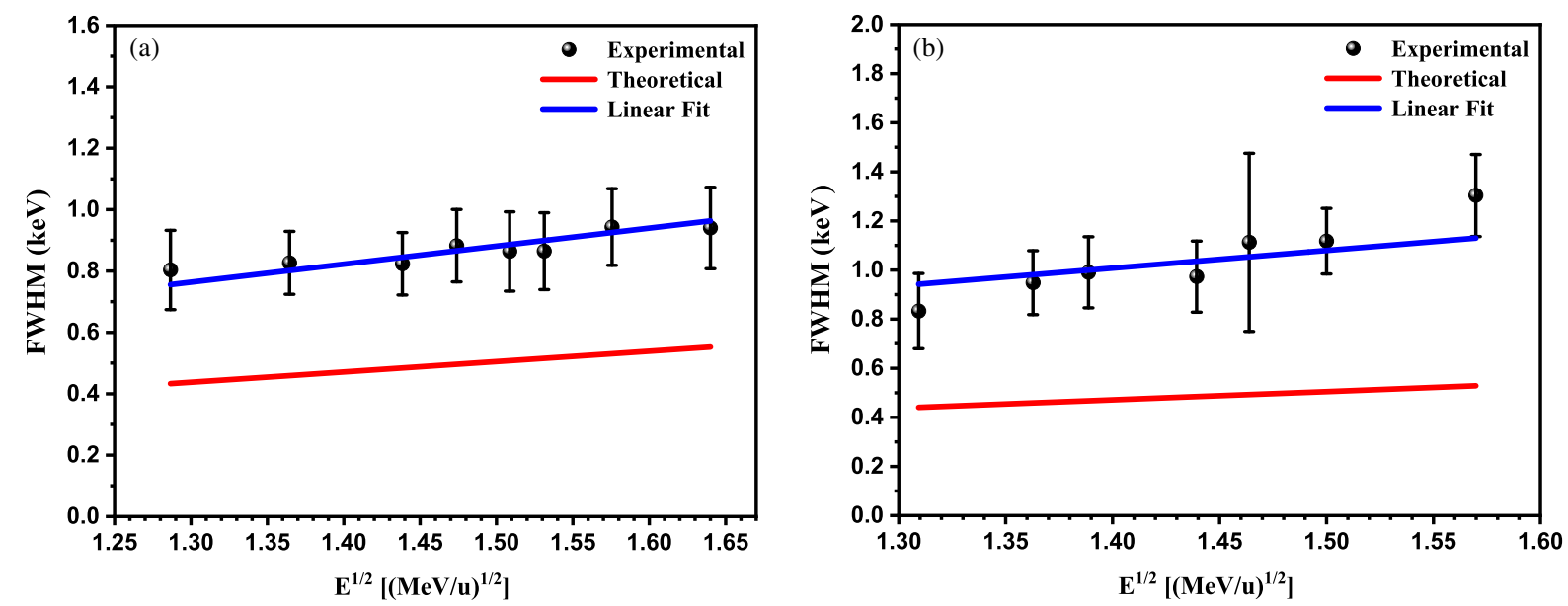

FIG. 3. Full width at half maximum $(\mathrm{FWHM})$ versus $\mathrm{E}^{1 / 2}\left[(\mathrm{MeV} / \mathrm{u})^{1 / 2}\right]$ : Variation of measured FWHM with respect to $\mathrm{E}^{1 / 2}$ (a) ${ }^{58} \mathrm{Ni}$ on ${ }^{12} \mathrm{C}$ and (b) ${ }^{56} \mathrm{Fe}$ on ${ }^{12} \mathrm{C}$. Further, the measured values are compared with the theoretically calculated values of FWHM for the case of the H-like ion.

statistical error of the measured FWHM. Discrete x-ray lines including the REC peak appear on a continuous background originating from bremsstrahlung of the projectile ions. This background is exponential in nature and thus, every spectrum is fitted using a standard routine with an exponential background as shown in Fig. 1 with a dashed line. The REC peak appears at the end of this background label and thus, very small background existing is subtracted in the fitting procedure. Though no $\mathrm{x}$-ray lines are known to come into view at this region, no appreciable systematic error is present here. However, low statistics for the REC peaks do not allow us to ignore the systematic errors fully. Accordingly, the systematic error is kept within $5 \%$ as assumed in our earlier works [31,32]. The measured values of FWHMs with errors are plotted as a function of $\mathrm{E}^{1 / 2}$ as shown in Fig. 3. It is seen that the experimental values are substantially larger than the theoretical predictions. The difference can well be attributed to the overlapping contributions from the different K-REC peak energies originating from various charge states produced in the ion-solid collisions prior to the occurrence of REC process. Important to note that the K-shell binding energy (for the present case, $\triangle B_{P}=100-1000 \mathrm{eV}$ ) varies with the charge state of the projectile ions, which in turn changes the K-REC photopeak energy [Eq. (1)] and each peak is equally broadened with a width as obtained from Eq. (2).

The measured K-REC centroid energy versus the projectile energy is shown in Fig. 4. Large FWHM due to low statistics introduces large $(1 \%-2 \%)$ uncertainty in the centroid also. As expected, from Eq. (1), the variation of the K-REC energy shows a linear dependence on the increasing projectile energy. It is worth mentioning that
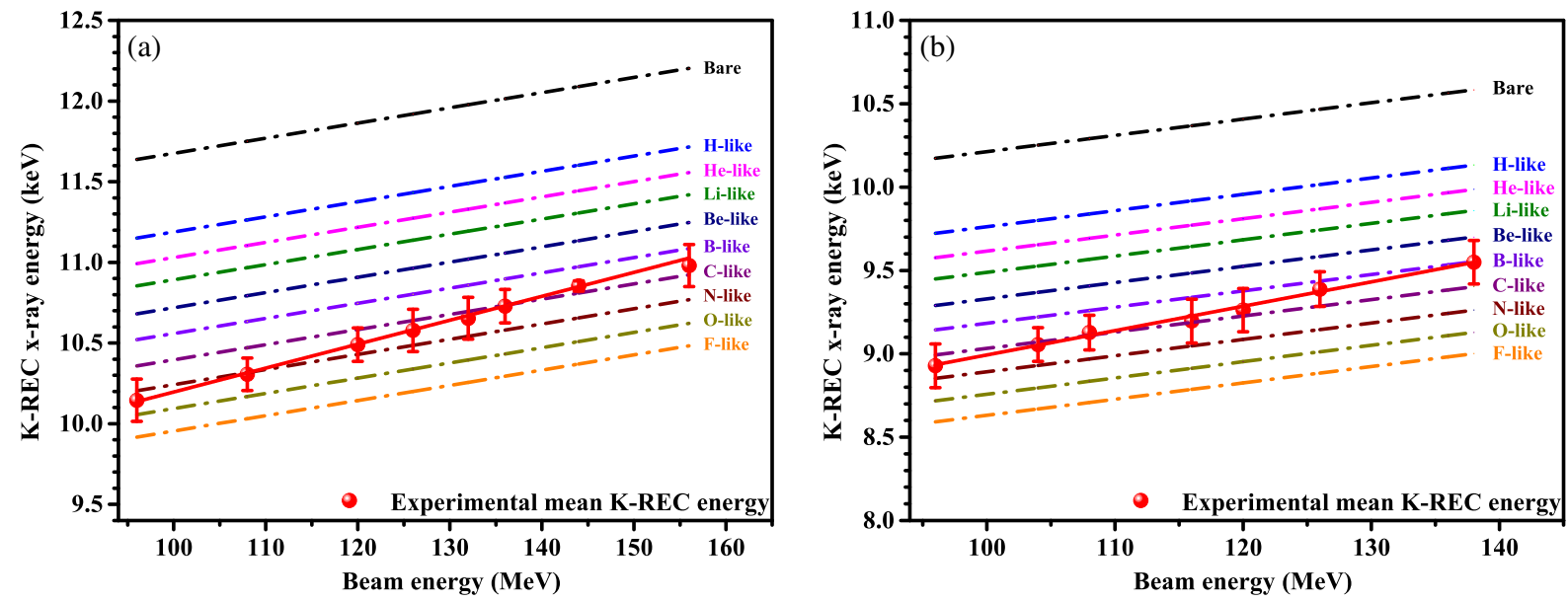

FIG. 4. K-REC $\mathrm{x}$-ray energy versus beam energy: Variation of K-REC peak centroid energy with the beam energy for (a) ${ }^{58} \mathrm{Ni}$ on ${ }^{12} \mathrm{C}$ and (b) ${ }^{56} \mathrm{Fe}$ on ${ }^{12} \mathrm{C}$ for various beam energies. The variation of theoretically calculated K-REC energy for different charge states of the projectile ion is also shown for comparison. 

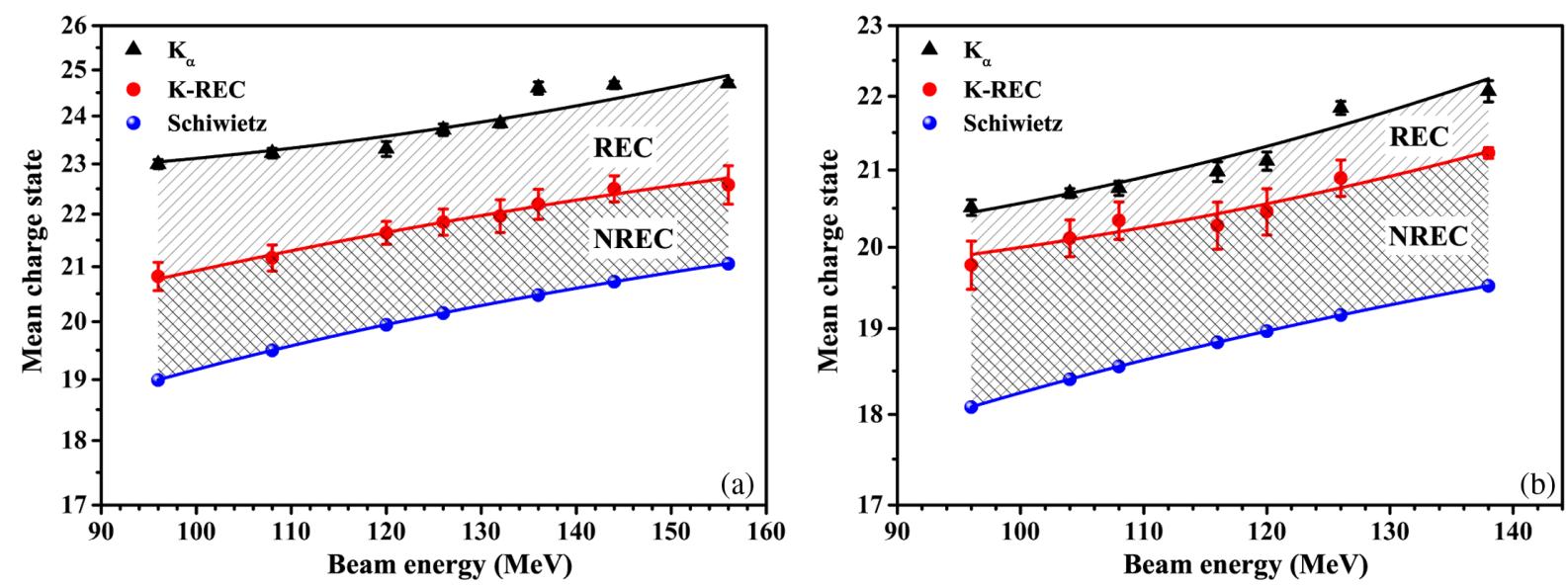

FIG. 5. Mean charge state versus beam energy: Comparison between present measurements (K-REC), earlier measurements $\left(\mathrm{K}_{\alpha}\right)$ [31], and Schiwietz formalism [34] predictions of mean charge state for the case of (a) ${ }^{58} \mathrm{Ni}$ on ${ }^{12} \mathrm{C}$ and (b) ${ }^{56} \mathrm{Fe}$ on ${ }^{12} \mathrm{C}$. Error bars are smeared within the symbol size.

the shift of K-REC centroid energy also depends on the vacancies created in the L-shell of the projectile ions during the collisions. Further, the measured K-REC energy is compared with the theoretically calculated K-REC energy for various projectile charge states created during the ionsolid collisions. The required K-shell binding energies corresponding to the different projectile charge states have been calculated with the multiconfiguration Dirac-Fock method using GRASP2K code [42].

Having studied the basic properties of the REC process through the K-REC x-ray energy and FWHM, we proceed to the main objective of the present work, i.e., to obtain the mean charge state of the projectile ion during the occurrence of the K-REC process. Knowing the binding energy of the target electron in the initial state (L-shell of the carbon atom), the binding energy of the final state (K-shell of the projectile ion with various vacancies in higher shells), where the target electron is going to be captured, has been calculated using Eq. (1), as all other factors like projectile mass and energy, are known. The $\mathrm{K}$-shell binding energy of the projectile ion so calculated represents the resultant binding energies of the various projectile charge states involved and can be defined as the mean K-shell binding energy of the projectile ions. Next, the measured mean K-shell binding energies of the projectile ions, which explicitly define the electronic configuration of the projectile ions, are used to calculate the mean charge state of the projectile ions. The corresponding mean charge state of the projectile ion is approximated by the interpolation of the theoretically calculated binding energy for the various charge states. The results obtained for mean charge states are portrayed in Fig. 5. The uncertainties in the mean charge state measurements are calculated using the standard procedure of error propagation. The figure also displays the mean charge state evolved in the bulk only as measured using the characteristic $\mathrm{K}_{\alpha} \mathrm{x}$-ray transitions [31]. As expected and discussed earlier, the values of projectile mean charge states obtained from K-REC peak structures (present work) found to be lower than the mean charge states obtained from $\mathrm{K}_{\alpha}$ peak (earlier work [31]). The mean charge state measurements using the K-REC peak structures provide us an opportunity to examine the above results with an intermediate stage. We see a noticeable difference between the effect of only bulk as measured from the $\mathrm{K}_{\alpha}$ peak and the bulk plus the REC processes as done from K-REC peak. Note that the transition of the electrons from L-shell to K-shell leads to $\mathrm{K}_{\alpha}$ emission and thus no change in the ionic stage of the projectile ions can take place during this process. Whereas, filling of the $\mathrm{K}$-vacancies of the projectile ions by the target electrons either from the bulk or the exit surface gives rise to K-REC radiation. Since the electron comes from outside the projectile ion, the process changes the charge state by one unit. Exactly, the difference of about one unit charge is found for the Fe experiments. However, this difference for $\mathrm{Ni}$ experiments is quite unusual; it is nearly of 2 units. It thus indicates that another electron is possibly getting captured with the projectile ions in the L-shell during the K-REC process, which may be quite likely with increasing charge state of the projectile ions. Hence, further studies are very much required to reveal the trend in higher as well as lower regions and then inferring the underlying mechanisms.

Comparing these two data sets with the mean charge state values obtained from the electromagnetic technique is essential for elaborating the dynamic nature of chargechanging processes at different regions (entrance surface, bulk, and exit surface) of the ion-solid interaction. However, no experimental data are available in the literature for the present energy range. Therefore, we have compared the measured mean charge states (both $\mathrm{K}_{\alpha}$ and K-REC) with the predictions of the empirical formalism, e.g., Schiwietz formalism [34], shown in Fig. 5. At the present energy range, the difference between experimental 
$\left(\mathrm{K}_{\alpha}\right)$ and empirical (Schiwietz formalism) mean charge states is about 3.5 and 4 units in ${ }^{56} \mathrm{Fe}$ and ${ }^{58} \mathrm{Ni}$ cases, respectively. The comparison gives a peculiar picture ensuring a fact that the mean charge states of the projectile ions in the bulk is quite different from the mean charge states measured after the collision process, i.e., when both the bulk and surface are accounted.

Whereas, the distinction between the measurements through the K-REC peak and the empirical results discerns that the REC process is one of the important chargeexchange processes originating at the surface; the role of dynamic screening and wake effects may be vital. The processes at the exit surface of the foil may include the formation of excited projectile bound states as high as Rydberg states due to nonradiative electron capture and wake riding electron capture to circular Rydberg states $[13,14]$ occurred because of finite surface wake field produced right at the exit surface $[15,29]$. The electrons, captured at such high-lying Rydberg states, are quite longlived and therefore can be detected with the electromagnetic techniques. Earlier measurements at low energies [9] as well as at high energies [43-45] corroborate such facts.

Present measurements are potentially important to explain some interesting facts, for example, Frey et al. [46] have measured that the stopping power of $1 \mathrm{MeV} / \mathrm{u}$ ${ }^{58} \mathrm{Ni}$ ions vary considerably with the incident charge states. The measurement shows that the stopping power increases with the charge states. Though this trend is reproduced with the theoretical calculations, however, the measured results are much higher than the theoretical values [47]. Note that the theoretical predictions make use of the incident charge state values because of the fact that the post-collision charge states are equal to the incident charge states. The stopping power of the ions passing through the thin carbon foils mostly depends on the ion-solid interactions in the bulk of the foil as the stopping power at the solid surface is comparatively very small [15]. Hence, the charge state information in the bulk of the foil is essential to estimate the stopping power correctly at the bulk, and such information can be available from experiments as the present one.

\section{CONCLUSION}

In the present work, the REC process has been studied for multielectron projectile ions. The mean K-shell binding energies, FWHM, and mean charge states of ${ }^{56} \mathrm{Fe}$ and ${ }^{58} \mathrm{Ni}$ projectile ions passing through the carbon target have been determined using the K-REC peak in the energy range of $1.65-3.03 \mathrm{MeV} / \mathrm{u}$. The variation of the K-REC energy shows a linear dependence on the increasing projectile energy as expected. The large FWHM of the K-REC profiles in the present measurements is responsible for the FWHM contributions from the different REC peaks originating at different energies corresponding to various charge states produced in the ion-solid collisions. The measured mean charge state values are compared with the ones measured with the $\mathrm{K}_{\alpha}$ lines and the empirical values. A notable difference has been found in the comparison between these three sets. The difference between the present mean charge state values and that obtained from the $\mathrm{K}_{\alpha}$ measurements is due to the contributions from the bulk and REC processes, whereas the difference between the empirical and the present mean charge state values can be ascribed to the contributions from nonradiative electron capture processes at the exit surface in the influence of dynamic screening and wake potential. It is worth mentioning that the electrons are captured at the long-lived Rydberg states, so that radiative or autoionizing decay does not take place before they are detected with the electromagnetic techniques. The significant difference in the mean charge state values for all three cases provides a clear indication that the charge-changing processes at different regions are disentangled and the charge state in the bulk is much larger than the charge state as analyzed with the electromagnetic technique. This fact explains well the difference between the experiment and theory on the charge state dependent stopping power values $[46,47]$.

\section{ACKNOWLEDGMENTS}

We would like to acknowledge the co-operation and support received from the Pelletron accelerator staff and all colleagues of atomic physics group, IUAC. We are grateful to Debasis Mitra for illuminating the discussion. PS is thankful to UGC, India for providing the fellowship as financial support to carry out this work.

[1] G. H. Henderson, Changes in the charge of an $\alpha$-particle passing through matter, Proc. R. Soc. London, Ser. A 102, 496 (1923).

[2] K. Ishii, A. Itoh, and K. Okuno, Electron-capture cross sections of multiply charged slow ions of carbon, nitrogen, and oxygen in He, Phys. Rev. A 70, 042716 (2004).

[3] P. Scharrer, C. E. Düllmann, W. Barth, J. Khuyagbaatar, A. Yakushev, M. Bevcic, P. Gerhard, L. Groening, K. P. Horn, E. Jäger, J. Krier, and H. Vormann, Measurements of charge state distributions of 0.74 and $1.4 \mathrm{MeV} / \mathrm{u}$ heavy ions passing through dilute gases, Phys. Rev. Accel. Beams 20, 043503 (2017).

[4] H.-D. Betz, Charge states and charge-changing cross sections of fast heavy ions penetrating through gaseous and solid media, Rev. Mod. Phys. 44, 465 (1972).

[5] H. W. Schnopper, H. D. Betz, J. P. Delvaille, K. Kalata, A. R. Sohval, K. W. Jones, and H. E. Wegner, Evidence for Radiative Electron Capture by Fast, Highly Stripped Heavy Ions, Phys. Rev. Lett. 29, 898 (1972).

[6] U. Wille and R. Hippler, Mechanisms of inner-shell vacancy production in slow ion-atom collisions, Phys. Rep. 132, 129 (1986).

[7] C. R. Vane, I. A. Sellin, M. Suter, G. D. Alton, S. B. Elston, P. M. Griffin, and R. S. Thoe, Z, Velocity, and Charge Dependence of Zero-Degree Electron "Cusps" from 
Charge Transfer to Continuum States of Bare and Highly Ionized Projectiles, Phys. Rev. Lett. 40, 1020 (1978).

[8] D. Schneider, D. DeWitt, A. S. Schlachter, R. E. Olson, W. G. Graham, J. R. Mowat, R. D. DuBois, D. H. Loyd, V. Montemayor, and G. Schiwietz, Strong continuumcontinuum couplings in the direct ionization of $\mathrm{Ar}$ and $\mathrm{He}$ atoms by $6-\mathrm{MeV} / \mathrm{u} \mathrm{U}^{38+}$ and $\mathrm{Th}^{38+}$ projectiles, Phys. Rev. A 40, 2971 (1989).

[9] S. Martin, A. Denis, Y. Ouerdane, A. Salmoun, A. El Motassadeq, J. Désesquelles, M. Druetta, D. Church, and T. Lamy, Multielectron Capture in $\mathrm{Kr}^{18+}$ Collisions with $\mathrm{Kr}$ and $\mathrm{Ar}$ at Low Energies by Rydberg Transition Spectroscopy, Phys. Rev. Lett. 64, 2633 (1990).

[10] M. Nofal et al., Radiative Electron Capture to the Continuum and the Short-Wavelength Limit of ElectronNucleus Bremsstrahlung in $90 A \mathrm{MeV} \mathrm{U}^{88+}\left(1 s^{2} 2 s^{2}\right)+$ $\mathrm{N}_{2}$ Collisions, Phys. Rev. Lett. 99, 163201 (2007).

[11] E. De Filippo, G. Lanzano, H. Rothard, and C. Volant, Three-Electron Auger Process from Beam-Foil Excited Multiply Charged Ions, Phys. Rev. Lett. 100, 233202 (2008).

[12] A. Simon, A. Warczak, T. ElKafrawy, and J. A. Tanis, Radiative Double Electron Capture in Collisions of $\mathrm{O}^{8+}$ Ions with Carbon, Phys. Rev. Lett. 104, 123001 (2010).

[13] M. Day and M. Ebel, Wake-bound states: Dispersive and surface effects, Phys. Rev. B 19, 3434 (1979).

[14] T. Nandi, Formation of the circular rydberg states in ionsolid collisions, Astrophys. J. 673, L103 (2008).

[15] T. Nandi, K. Haris, G. Singh, P. Kumar, R. Kumar, S. Saini, S. Khan, A. Jhingan, P. Verma, A. Tauheed, D. Mehta, and H. Berry, Fast Ion Surface Energy Loss and Straggling in the Surface Wake Fields, Phys. Rev. Lett. 110, 163203 (2013).

[16] S. K. Allison, Experimental results on charge-changing collisions of hydrogen and helium atoms and ions at kinetic energies above $0.2 \mathrm{kev}$, Rev. Mod. Phys. 30, 1137 (1958).

[17] H. D. Betz, in Atomic Physics Accelerators, Methods in Experimental Physics, edited by P. Richard (Academic Press, New York, 1980), Vol. 17, pp. 73-148.

[18] A. B. Wittkower and H. D. Betz, Equilibrium-charge-state distributions of energetic ions $(Z>2)$ in gaseous and solid media, Atomic Data and Nuclear Data Tables 5, 113 (1973).

[19] K. Shima, T. Mikumo, and H. Tawara, Equilibrium charge state distributions of ions $\left(Z_{1} \geq 4\right)$ after passage through foils: Compilation of data after 1972, At. Data Nucl. Data Tables 34, 357 (1986).

[20] K. Shima, N. Kuno, M. Yamanouchi, and H. Tawara, Equilibrium charge fractions of ions of $Z=4-92$ emerging from a carbon foil, At. Data Nucl. Data Tables 51, 173 (1992).

[21] H. Okuno, N. Fukunishi, A. Goto, H. Hasebe, H. Imao, O. Kamigaito, M. Kase, H. Kuboki, Y. Yano, S. Yokouchi, and A. Hershcovitch, Low-Z gas stripper as an alternative to carbon foils for the acceleration of highpower uranium beams, Phys. Rev. ST Accel. Beams 14, 033503 (2011).

[22] H. Imao, H. Okuno, H. Kuboki, S. Yokouchi, N. Fukunishi, O. Kamigaito, H. Hasebe, T. Watanabe, Y. Watanabe,
M. Kase, and Y. Yano, Charge stripping of ${ }^{238} \mathrm{U}$ ion beam by helium gas stripper, Phys. Rev. ST Accel. Beams 15, 123501 (2012).

[23] H. Kuboki, H. Okuno, H. Hasebe, N. Fukunishi, E. Ikezawa, H. Imao, O. Kamigaito, and M. Kase, Charge state distribution of ${ }^{86} \mathrm{Kr}$ in hydrogen and helium gas charge strippers at 2.7 MeV/nucleon, Phys. Rev. ST Accel. Beams 17, 123501 (2014).

[24] R. N. Sagaidak, N. A. Kondratiev, L. Corradi, E. Fioretto, T. Mijatovic, G. Montagnoli, F. Scarlassara, A. M. Stefanini, and S. Szilner, Charge distributions of Ra recoil ions produced in ${ }^{12} \mathrm{C}+\mathrm{Pb}$ fusion-evaporation reactions, Phys. Rev. C 97, 054622 (2018).

[25] C. J. Schmitt, Equilibrium charge state distributions of low-Z ions incidents on thin self-supporting foils, Ph.D. thesis, University of Notre Dame, 2010.

[26] M. Imai, M. Sataka, K. Kawatsura, K. Takahiro, K. Komaki, H. Shibata, H. Sugai, and K. Nishio, Equilibrium and non-equilibrium charge-state distributions of $2 \mathrm{MeV} / u$ sulfur ions passing through carbon foils, Nucl. Instrum. Methods Phys. Res., Sect. B 267, 2675 (2009).

[27] M. Imai, M. Sataka, M. Matsuda, S. Okayasu, K. Kawatsura, K. Takahiro, K. Komaki, H. Shibata, and K. Nishio, Equilibrium and non-equilibrium charge-state distributions of $2.0 \mathrm{MeV} / u$ carbon ions passing through carbon foils, Nucl. Instrum. Methods Phys. Res., Sect. B 354, 172 (2015).

[28] N. Novikov and Y. A. Teplova, Methods of estimation of equilibrium charge distribution of ions in solid and gaseous media, Phys. Lett. A 378, 1286 (2014).

[29] N. janan Al-Bahnam, K. A. Ahmad, and A. I. A. AlNuman, Wake potential of swift ion in amorphous carbon target, Phys. Lett. A 381, 616 (2017).

[30] J. Burgdörfer, Dynamic screening and wake effects on electronic excitation in ion-solid and ion-surface collisions, Nucl. Instrum. Methods Phys. Res., Sect. B 67, 1 (1992).

[31] P. Sharma and T. Nandi, X-Ray spectroscopy: An experimental technique to measure charge state distribution during ion-solid interaction, Phys. Lett. A 380, 182 (2016).

[32] P. Sharma and T. Nandi, Experimental evidence of beamfoil plasma creation during ion-solid interaction, Phys. Plasmas 23, 083102 (2016).

[33] P. Sharma and T. Nandi, Shakeoff Ionization near the Coulomb Barrier Energy, Phys. Rev. Lett. 119, 203401 (2017).

[34] G. Schiwietz and P. L. Grande, Improved charge-state formulas, Nucl. Instrum. Methods Phys. Res., Sect. B 175-177, 125 (2001).

[35] J. Eichler and T. Stöhlker, Radiative electron capture in relativistic ion-atom collisions and the photoelectric effect in hydrogen-like high-z systems, Phys. Rep. 439, 1 (2007).

[36] J. Lindskog, J. Phil, R. Sjödin, A. Marelius, K. Sharma, R. Hallin, and P. Lindner, Radiative electron capture by multiply-ionized chlorine atoms, Phys. Scr. 14, 100 (1976).

[37] R. Schule, H. Schmidt-Bocking, and I. Tserruya, Radiative electron capture by ${ }^{32}$ S ions, J. Phys. B 10, 889 (1977).

[38] P. Kienle, M. Kleber, B. Povh, R. M. Diamond, F. S. Stephens, E. Grosse, M. R. Maier, and D. Proetel, Radiative Capture and Bremsstrahlung of Bound Electrons Induced by Heavy Ions, Phys. Rev. Lett. 31, 1099 (1973). 
[39] H. Tawara, P. Richard, and K. Kawatsura, Radiative electroncapture processes in zero- and one-electron heavy-ion collisions with He, Phys. Rev. A 26, 154 (1982).

[40] J. A. Tanis, S. M. Shafroth, J. E. Willis, and J. R. Mowat, Mean $K$-shell binding energies for highly stripped chlorine ions determined from radiative electron-capture energies, Phys. Rev. A 27, 1671 (1983).

[41] S. Reed and N. Ware, The measurement of X-ray peak intensities with a lithium drifted silicon detector by integration over a fixed energy range, J. Phys. E 5, 1112 (1972).

[42] P. Jönsson, X. He, C. Froese Fischer, and I. Grant, The GRASP2K relativistic atomic structure package, Comput. Phys. Commun. 177,597 (2007).

[43] A. Müller, B. Schuch, W. Groh, and E. Salzborn, Multipleelectron processes in $1.4 \mathrm{MeV} / \mathrm{u}$ ion-atom collisions, $\mathrm{Z}$. Phys. D 7, 251 (1987).
[44] A. S. Schlachter, J. W. Stearns, K. H. Berkner, E. M. Bernstein, M. W. Clark, R. D. DuBois, W. G. Graham, T. J. Morgan, D. W. Mueller, M. P. Stockli, J. A. Tanis, and W. T. Woodland, in Sixteenth International Conference on the Physics of Electron and Atomic Collisions (New York, 1989), pp. 16.

[45] H. Bräuning et al., Strong Evidence for Enhanced Multiple Electron Capture from Surfaces in $46 \mathrm{MeV} / u \mathrm{~Pb}^{81+}$ Collisions with Thin Carbon Foils, Phys. Rev. Lett. 86, 991 (2001).

[46] C. Frey, G. Dollinger, A. Bergmaier, T. Faestermann, and P. Maier-Komor, Charge state dependence of the stopping power of $1 \mathrm{MeV} / A^{58} \mathrm{Ni}$ ions, Nucl. Instrum. Methods Phys. Res., Sect. B 107, 31 (1996).

[47] P. Sigmund, Charge-dependent electronic stopping of swift nonrelativistic heavy ions, Phys. Rev. A 56, 3781 (1997). 